\title{
Uso de ultrasonido pulmonar para predecir mortalidad intrahospitalaria en pacientes con infección por COVID-19
}

\author{
Daniel Manzur-Sandoval, ${ }^{1}$ Edgar García-Cruz, ${ }^{1}$ Rodrigo Gopar-Nieto, ${ }^{2 *}$ Diego Araiza-Garaygordobil, ${ }^{2}$ \\ Arturo Garza-de la Maza, ${ }^{1}$ Edith Ramírez-Lara, ${ }^{1}$ Rodrigo Zebadua-Torres, ${ }^{3}$ Ricardo L. Barajas-Campos, ${ }^{4}$ \\ Rafael Rascón-Sabido, ${ }^{5}$ Gastón Mendoza-Copa, ${ }^{6}$ Esteban I. Chango-Criollo, ${ }^{7}$ Gabriela Ramírez-Galindo ${ }^{1}$ \\ y Gustavo Rojas-Velasco ${ }^{1}$ \\ ${ }^{1}$ Unidad de Cuidados Intensivos Cardiovasculares; ${ }^{2}$ Unidad de Cuidados Coronarios; ${ }^{3}$ Departamento Cardiopulmonar; ${ }^{4}$ Servicio de Hospitalización; \\ ${ }^{5}$ Hospital Naval de Especialidades de Veracruz, Servicio de Insuficiencia Cardiaca, Veracruz, México; ${ }^{6}$ Hospital de Clínicas, Unidad de Cuidados \\ Intensivos, La Paz, Bolivia; ${ }^{7}$ Hospital General Docente "Vicente Corral Moscoso", Unidad de Cuidados Intensivos, Cuenca, Ecuador. ${ }^{1-4}$ Instituto \\ Nacional de Cardiología "Ignacio Chávez", Ciudad de México, México.
}

\section{Resumen}

Introducción: La implementación del ultrasonido pulmonar (LUS) en los pacientes con COVID-19 puede ayudar a establecer el grado de afectación pulmonar, evaluar la respuesta al tratamiento y estimar el desenlace intrahospitalario. Objetivo: Evaluar la aplicación de un protocolo LUS en pacientes con infección por COVID-19 para predecir mortalidad intrahospitalaria. Métodos: El estudio se realizó del 1 de abril al 1 de agosto de 2020 en pacientes con infección por COVID-19, ingresados en la Unidad de Terapia Intensiva. Se realizó evaluación pulmonar por médicos entrenados en ultrasonografía crítica. Resultados: La mayoría de los pacientes fue del sexo masculino, la edad mediana fue de 56 años y 59 \% requirió ventilación mecánica. La mortalidad intrahospitalaria fue de $39.4 \%$ y en aquellos con puntuación de LUS $\geq 19$, de 50 \%. El modelo de regresión logística múltiple mostró que la puntuación de LUS $\geq 19$ se asoció significativamente a mortalidad (cociente de riesgo $=2.55$, $p=0.01$ ). Conclusiones: El LUS es una herramienta clínica segura y rápida que puede realizarse al lado de la cama de los pacientes con infección por COVID-19, para establecer el grado de afectación parenquimatosa y predecir la mortalidad.

PALABRAS CLAVE: COVID 19. Ecografía pulmonar. Mortalidad.

\section{Use of pulmonary ultrasound to predict in-hospital mortality in patients with COVID-19 infection}

\begin{abstract}
Introduction: Lung ultrasound (LUS) implementation in patients with COVID-19 can help to establish the degree of pulmonary involvement, evaluate treatment response and estimate in-hospital outcome. Objective: To evaluate the application of LUS in patients with COVID-19 infection to predict in-hospital mortality. Methods: The study was carried out from April 1 to August 1 , 2020 in patients with COVID-19 infection admitted to the Intensive Care Unit. Lung evaluation was carried out by physicians trained in critical care ultrasonography. Results: Most patients were males, median age was 56 years, and $59 \%$ required mechanical ventilation. In-hospital mortality was $39.4 \%$, and in those with a LUS score $\geq 19$, mortality was higher (50\%). The multiple logistic regression model showed that a LUS score $\geq 19$ was significantly associated with mortality (hazard ratio $=2.55, p=0.01$ ). Conclusions: LUS is a safe and fast clinical tool that can be applied at bedside in patients with COVID-19 infection to establish the degree of parenchymal involvement and predict mortality.
\end{abstract}

KEY WORDS: COVID 19. Lung ultrasound. Mortality.

Correspondencia:

*Rodrigo Gopar-Nieto

E-mail: rodrigogopar@gmail.com

DOI: 10.24875/GMM.20000768
Gac Med Mex. 2021:157:261-266

Disponible en PubMed

www.gacetamedicademexico.com

0016-3813/@ 2021 Academia Nacional de Medicina de México, A.C. Publicado por Permanyer. Este es un artículo open access bajo la licencia CC BY-NC-ND (http://creativecommons.org/licenses/by-nc-nd/4.0/). 


\section{Introducción}

EI SARS-CoV-2 es un agente que apareció en diciembre de 2019 y que causa COVID-19. ${ }^{1}$ Pertenece al mismo grupo de virus ARN que ocasionan enfermedades respiratorias graves como SARS y MERS. ${ }^{2}$ La principal manifestación de la infección por SARSCoV-2 es una neumonía viral que puede evolucionar a síndrome de dificultad respiratoria aguda grave; por lo tanto, el brote de este virus demanda más hospitalizaciones en urgencias y unidades de cuidados críticos, con una alta proporción de requerimiento de ventilación mecánica.

El uso de la ultranosografía pulmonar (LUS) como herramienta diagnóstica en pacientes críticos para establecer el grado de afectación parenquimatosa, evaluar la respuesta al tratamiento y durante el seguimiento es una práctica común que se ha convertido en un estándar de alta calidad para la atención al lado de la cama del paciente. ${ }^{3,4}$

Múltiples estudios han descrito que la tomografía axial computarizada y el LUS muestran una importante correlación con los principales síndromes pulmonares, como los síndromes intersticiales, el neumotórax, la consolidación pulmonar y el derrame pleural. ${ }^{5}$ La implementación del LUS puede disminuir las solicitudes de radiografía de tórax y tomografía computarizada, lo cual es particularmente relevante para mejorar las políticas de seguridad hospitalaria con el fin de limitar la exposición del personal al de SARS-CoV-2. ${ }^{6}$

A pesar del tiempo transcurrido desde el primer caso de COVID-19 en Wuhan, China, los grupos de trabajo de todo el mundo están tratando de responder preguntas sobre cómo los protocolos pueden aumentar el poder diagnóstico del LUS y proporcionar elementos predictivos de resultados hospitalarios. ${ }^{7}$ Un estudio unicéntrico reciente mostró que la observación en el LUS de una mayor afectación predice mortalidad y la necesidad de ventilación mecánica; ${ }^{8}$ sin embargo, esos hallazgos no se han probado en otros lugares. El objetivo de este estudio fue evaluar la utilidad de un protocolo de LUS en pacientes con infección por COVID-19 para predecir la mortalidad intrahospitalaria.

\section{Método}

Este estudio transversal incluyó a 101 pacientes adultos con infección confirmada por COVID-19 (mediante RT-PCR, reacción en cadena de la polimerasa con transcripción inversa en tiempo real para detección de ARN del SARS-CoV-2), ingresados en la Unidad de Cuidados Intensivos del Instituto Nacional de Cardiología "Ignacio Chávez", Ciudad de México, entre el 1 de abril y el 15 de agosto de 2020. La evaluación se realizó mediante la técnica de LUS indicada en el protocolo ORACLE. ${ }^{4}$ El comité institucional del Instituto aprobó la investigación. El paciente 0 un representante legalmente autorizado proporcionó el consentimiento informado por escrito.

\section{Adquisición y cuantificación de imágenes}

El protocolo fue diseñado para permitir la adquisición rápida de imágenes al lado de la cama del paciente y el análisis de la imagen se realizó fuera de su habitación. El estudio fue realizado por cardiólogos y especialistas en cuidados críticos con experiencia en grabación e interpretación de ultrasonido en pacientes en estado crítico. En todos los casos, la evaluación completa del LUS se realizó al ingreso del paciente a la Unidad de Cuidados Intensivos en menos de cinco minutos. Se aplicaron las siguientes medidas para minimizar la exposición del personal a la infección:

- Todos los estudios a los pacientes se realizaron durante las rondas regulares o de rutina.

- La máquina y la sonda sectorial se desinfectaron adecuadamente entre cada evaluación.

- Todo el personal tuvo equipo de protección personal adecuado.

- Las mediciones se realizaron fuera de la habitación del paciente.

El operador obtuvo las imágenes con una sonda sectorial en fase, a 2 o $3 \mathrm{mHz}$, desde el lado derecho o izquierdo del paciente, en cualquier plataforma y con los modos $\mathrm{M}$ y 2D, con una profundidad de $10 \mathrm{a}$ $15 \mathrm{~cm}$. En cada hemitórax se evaluaron los siguientes puntos, comenzando por el lado derecho y con el marcador de la sonda hacia la cabeza del paciente:

- Anterosuperior: segundo espacio intercostal, línea medioclavicular (puntos 1 y 7 ).

- Anteroinferior: quinto espacio intercostal, línea medioclavicular (puntos 2 y 8 ).

- Laterosuperior: segundo espacio intercostal, línea axilar anterior (puntos 3 y 9 ).

- Lateroinferior: quinto espacio intercostal, línea axilar anterior (puntos 4 y 10).

- Posterosuperior: segundo espacio intercostal, línea axilar media a posterior (puntos 5 y 11).

- Posteroinferior: quinto espacio intercostal, línea axilar media a posterior (puntos 6 y 12). 


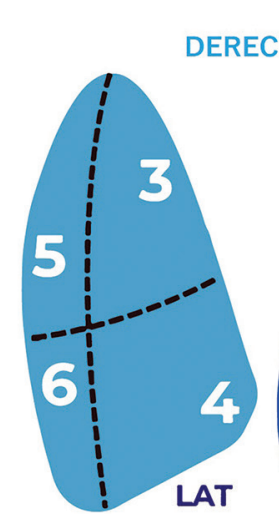

POST

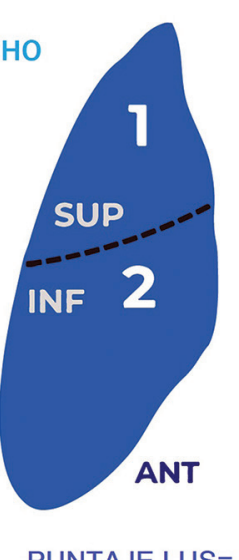

PUNTAJE LUS= $19(16-22)$

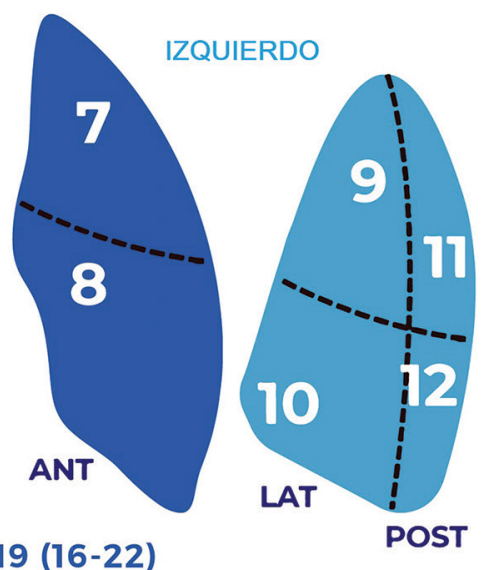

POST

\begin{tabular}{lllllllllllll}
\hline Zona LUS & 1 & $\mathbf{2}$ & $\mathbf{3}$ & $\mathbf{4}$ & $\mathbf{5}$ & $\mathbf{6}$ & $\mathbf{7}$ & $\mathbf{8}$ & $\mathbf{9}$ & 10 & 11 & $\mathbf{1 2}$ \\
\hline Hallazgo & Perfil B & Perfil B & Perfil B & Perfil B & Perfil B & Perfil B & Perfil B & Perfil B & Perfil B & Perfil B & Perfil B & Perfil B \\
más & $>3 \mathrm{~mm}$ & $>3 \mathrm{~mm}$ & $<3 \mathrm{~mm}$ & $<3 \mathrm{~mm}$ & $<3 \mathrm{~mm}$ & $>3 \mathrm{~mm}$ & $>3 \mathrm{~mm}$ & $>3 \mathrm{~mm}$ & $<3 \mathrm{~mm}$ & $>3 \mathrm{~mm}$ & $<3 \mathrm{~mm}$ & $<3 \mathrm{~mm}$ \\
común & $40.6 \%$ & $47.5 \%$ & $46.5 \%$ & $47.5 \%$ & $45.5 \%$ & $42.6 \%$ & $46.5 \%$ & $49.5 \%$ & $53.5 \%$ & $41.6 \%$ & $45.5 \%$ & $46.5 \%$ \\
& & & & & & & & & & & & \\
\hline
\end{tabular}

Figura 1. Puntos de exploración y los hallazgos más comunes en cada región.

En cada punto se aplicaron las siguientes puntuaciones y se calculó la suma total de los 12 puntos (Figura 1):

- 0, perfil A: presencia de líneas A con menos de tres líneas $B$.

- 1, perfil B: presencia de tres o más líneas $B$ con separación $>3 \mathrm{~mm}$.

- 2, perfil B: presencia de tres o más líneas $B$ con separación $\leq 3 \mathrm{~mm}$.

- 3: perfil C: consolidación, señal de trituración o perfil A/B. ${ }^{4}$

Para determinar la puntuación RALE, cada radiografía se dividió en cuadrantes, definidos verticalmente por la columna vertebral y horizontalmente por la primera rama del bronquio principal izquierdo. A cada cuadrante se le asignó una puntuación de consolidación de 0 a 4 para cuantificar la extensión de las opacidades alveolares. Para calcular la puntuación RALE final, se sumó el producto de la puntuación de consolidación de cada cuadrante. ${ }^{9} \mathrm{La}$ puntuación RALE fue calculada por un especialista en medicina cardiopulmonar.

\section{Análisis estadístico}

Para las variables continuas se utilizó la prueba de normalidad de Shapiro-Wilk; después de conocer su distribución fueron registradas con media y desviación estándar si eran paramétricas, y mediana y rango intercuartilar (RIC) si eran no paramétricas. Las comparaciones entre las variables continuas se realizaron con la prueba $U$ de Mann-Whitney. Las variables categóricas fueron descritas con frecuencias y porcentajes; para las comparaciones se utilizaron las pruebas $\chi^{2}$ y exacta de Fisher, según correspondiera conforme a los valores esperados.

Con el fin de establecer un punto de corte para las líneas B, utilizamos el análisis ROC, en el que 19 o más líneas $B$ arrojaron la mayor sensibilidad, especificidad y probabilidad predictiva. Realizamos un modelo de regresión logística, ajustado por edad y sexo, para conocer las variables que predecían la mortalidad. Con gráficos de Kaplan-Meier se comparó la mortalidad entre los grupos. Todos los análisis estadísticos se consideraron significativos con un valor de $p<0.05$. El análisis estadístico se realizó con STATA versión 14.

\section{Resultados}

Se recopilaron datos clínicos de 101 pacientes consecutivos con infección confirmada por COVID-19.

La mayoría de los pacientes fueron hombres $(76.2 \%)$, con una mediana de edad de $56(\mathrm{RIC}=48-65)$ años, y las comorbilidades más frecuentes fueron la hipertensión y la diabetes tipo 2 (38 y 33 \%, respectivamente). La mediana de la presión arterial de oxígeno/fracción inspirada de oxigeno $\left(\mathrm{PaO}_{2} / \mathrm{FiO}_{2}\right)$ fue 190. Los pacientes estuvieron hemodinámicamente estables al ingreso, con una mediana de la frecuencia 
Tabla 1. Características clínicas y demográficas

\begin{tabular}{|c|c|c|c|c|c|c|c|}
\hline \multirow[t]{3}{*}{ Variable } & \multirow{2}{*}{\multicolumn{2}{|c|}{ Total }} & \multicolumn{4}{|c|}{ Puntuación de LUS } & \multirow[t]{3}{*}{$p$} \\
\hline & & & \multicolumn{2}{|c|}{$<19$} & \multicolumn{2}{|c|}{$\geq 19$} & \\
\hline & $n$ & $(\%)$ & $n$ & $(\%)$ & $n$ & $(\%)$ & \\
\hline Hombres & 77 & 76.2 & 33 & 73.3 & 44 & 78.6 & 0.64 \\
\hline Mujeres & 24 & 23.8 & 12 & 26.7 & 12 & 21.4 & 0.64 \\
\hline Hipertensión & 38 & 37.6 & 19 & 42.2 & 19 & 33.9 & 0.39 \\
\hline Diabetes mellitus & 33 & 32.7 & 17 & 37.8 & 16 & 28.6 & 0.32 \\
\hline Tabaquismo & 30 & 29.7 & 16 & 35.6 & 14 & 25 & 0.24 \\
\hline \multirow[t]{2}{*}{ Dislipidemia } & 17 & 16.8 & 9 & 20 & 8 & 14.3 & 0.44 \\
\hline & Mediana & RIC & Mediana & RIC & Mediana & RIC & \\
\hline Edad, años & 56 & $48-65$ & 57 & $50-63$ & 56 & $47-66$ & 0.85 \\
\hline $\mathrm{PaO}_{2} / \mathrm{FiO}_{2}$ & 198 & $145-260$ & 200 & $156-260$ & 190 & $142-257$ & 0.75 \\
\hline - Frecuencia cardiaca, Ipm & 80 & $73-92$ & 80 & $75-88$ & 88 & $70-95$ & 0.24 \\
\hline - Tensión arterial sistólica, mm Hg & 125 & 110-134 & 128 & $110-133$ & 124 & 113-135 & 0.77 \\
\hline - Tensión arterial diastólica, mm Hg & 74 & $67-80$ & 75 & $65-80$ & 74 & $67-81$ & 0.76 \\
\hline $\begin{array}{l}\text { Troponina I al ingreso, } \mathrm{pg} / \mathrm{mL} \\
\text { (14-42.9 pg/mL) }\end{array}$ & 15 & $7-58$ & 22 & 7.7-73 & 14 & $6-44$ & 0.30 \\
\hline Troponina I máxima (pg/mL) & 93 & 23-265 & 123 & $24-354$ & 83 & 20-238 & 0.39 \\
\hline $\begin{array}{l}\text { Dímero D al ingreso }(\mathrm{ng} / \mathrm{mL}) \\
(<500 \mathrm{ng} / \mathrm{mL})\end{array}$ & 610 & $272-1732$ & 590 & $271-2588$ & 610 & $272-1420$ & 0.74 \\
\hline Dímero D máximo (ng/mL) & 1890 & $690-5620$ & 3555 & $690-8084$ & 1450 & $720-2855$ & 0.20 \\
\hline NT-proBNP al ingreso, pg/mL (15-125 pg/mL) & 759 & $220-3475$ & 716 & $299-4058$ & 900 & $205-3261$ & 0.97 \\
\hline NT-proBNP máximo, pg/mL & 1114 & $409-7004$ & 943 & $409-5367$ & 1186 & $339-7004$ & 0.77 \\
\hline Creatinina al ingreso, mg/dL & 0.9 & $0.7-1.45$ & 0.9 & $0.7-1.8$ & 0.9 & $0.6-1.2$ & 0.69 \\
\hline Creatinina máxima, mg/dL & 1.2 & $0.9-2.7$ & 1.1 & $0.9-2.9$ & 1.3 & $0.9-2.6$ & 0.79 \\
\hline Proteína $\mathrm{C}$ reactiva al ingreso, mg/dL (1-3 mg/L) & 128 & $61-246$ & 119 & $42-209$ & 136 & $66-262$ & 0.43 \\
\hline Proteína C reactiva máxima, mg/dL & 261 & $139-346$ & 239 & $137-321$ & 278 & 147-362 & 0.32 \\
\hline $\begin{array}{l}\text { Ferritina al ingreso, } \mathrm{mg} / \mathrm{dL} \\
(23.9-336 \mathrm{ng} / \mathrm{mL})\end{array}$ & 744 & 409-1306 & 784 & $430-1110$ & 667 & $366-1352$ & 0.91 \\
\hline Ferritina máxima, mg/dL & 1069 & $560-2000$ & 955 & $540-1470$ & 1224 & $656-2000$ & 0.13 \\
\hline Puntuación RALE & 6 & $4-7$ & 6 & $4-7$ & 6 & $4-7$ & 0.67 \\
\hline
\end{tabular}

$\mathrm{IRC}=$ rango intercuartilar NT-proBNP = péptido natriurético tipo N-terminal $\mathrm{B}$.

cardiaca de 80 (70-92) Ipm, presión arterial sistólica de 125 (110-134) mm Hg y presión arterial diastólica de $74(67-80) \mathrm{mm} \mathrm{Hg}$. Los estudios de laboratorio revelaron niveles elevados de la fracción $\mathrm{N}$-terminal del propéptido natriurético de tipo B (NT-proBNP), dímero $D$, proteína $C$ reactiva y ferritina al ingreso. $\mathrm{La}$ mediana de la puntuación RALE fue 6 (Tabla 1). El $59 \%$ de los pacientes requirió ventilación mecánica y la mortalidad hospitalaria fue de $39.4 \%$; en los pacientes con puntuación de LUS $\geq 19$, la mortalidad fue mayor (50\%) que en los que tuvieron puntuación < 19 (Tabla 2). En un subanálisis, únicamente la sobreinfección (cociente de riesgo $[\mathrm{HR}]=3.42, \mathrm{p}=0.001$ ) predijo la necesidad de ventilación mecánica.

Se documentó una puntuación mediana de LUS de 19 puntos. En la Figura 1 se representa el hallazgo ecográfico más frecuente en cada punto pulmonar. El modelo de regresión logística múltiple para la 
Tabla 2. Desenlaces intrahospitalarios

\begin{tabular}{|c|c|c|c|c|c|c|c|}
\hline \multirow[t]{3}{*}{ Variable } & \multirow{2}{*}{\multicolumn{2}{|c|}{ Total }} & \multicolumn{4}{|c|}{ Puntuación de LUS } & \multirow[t]{3}{*}{$\mathrm{p}$} \\
\hline & & & \multicolumn{2}{|c|}{$<19$} & \multicolumn{2}{|c|}{$\geq 19$} & \\
\hline & $\mathrm{n}$ & $\%$ & $n$ & $\%$ & $n$ & $\%$ & \\
\hline Ventilación mecánica & 59 & 58.4 & 22 & 48.9 & 37 & 66.1 & 0.10 \\
\hline Muerte & 39 & 39.4 & 12 & 26.7 & 27 & 50 & 0.01 \\
\hline \multirow[t]{2}{*}{ Otra infección } & 11 & 10.9 & 4 & 8.9 & 7 & 12.5 & 0.75 \\
\hline & Mediana & RIC & Mediana & RIC & Mediana & RIC & \\
\hline Días en ventilación mecánica & 6 & $0-15$ & 0 & $0-18$ & 8 & $0-14$ & 0.55 \\
\hline Días de hospitalización & 15 & $10-24$ & 17 & $11-26$ & 13 & $10-21$ & 0.20 \\
\hline
\end{tabular}

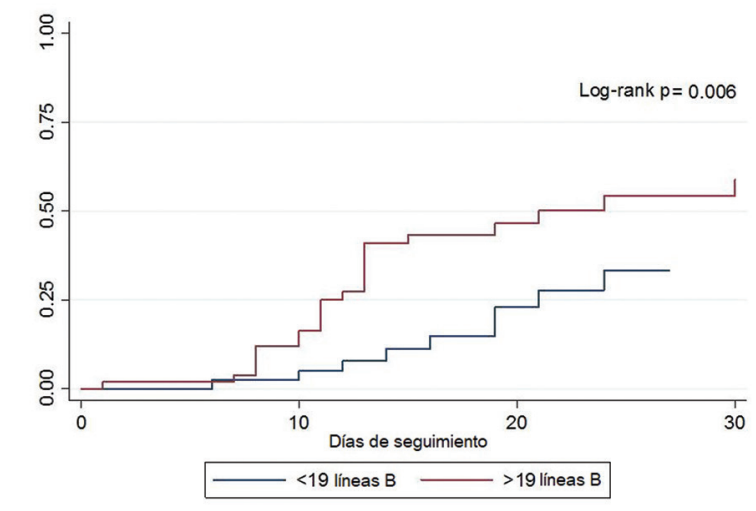

Figura 2. Gráfica de Kaplan-Meier en la que se compara la mortalidad entre pacientes conforme las puntuaciones de LUS.

predicción de la mortalidad mostró que la puntuación de LUS $\geq 19$ se asoció significativamente con el resultado ( $\mathrm{HR}=2.55, \mathrm{p}=0.01$ ), Tabla 3. En los pacientes con puntuación de LUS $\geq 19$, la mortalidad hospitalaria fue significativamente mayor $(\log$ rank $p=0.006)$ (Figura 2).

En el modelo de regresión logística múltiple, la ventilación mecánica $(H R=3.2, p=0.03)$, la troponina I $>20 \mathrm{pg} / \mathrm{mL}(\mathrm{HR}=2.1, \mathrm{p}=0.02$ ) ylaNT-proBNP $>800 \mathrm{pg} / \mathrm{mL}$ $(H R=2.55, p=0.02)$ predijeron mortalidad (Tabla 3).

\section{Discusión}

En nuestro estudio mostramos que una evaluación estructurada con LUS y el hallazgo de una puntuación elevada de este al ingreso hospitalario predijeron mortalidad en pacientes con COVID-19.

En cuanto a las variables clínicas, no hubo variación significativa entre la presencia de una puntuación de
LUS $\geq 19$ y los niveles de $\mathrm{PaO}_{2} / \mathrm{FiO}_{2}$, lo que sugiere que ya existe un grado significativo de afectación parenquimatosa pulmonar al ingreso antes de que las puntuaciones de oxigenación alcancen niveles críticos; la misma situación ocurre con la puntuación radiográfica RALE. El claro aumento de la mortalidad ante la mayor afectación parenquimatosa documentada mediante LUS sugiere que la identificación precoz de este hallazgo podría orientar al clínico a establecer un tratamiento más agresivo al ingreso. El LUS podría ayudar a prever los desenlaces e, incluso, la necesidad de trasladar al paciente tempranamente a un área de cuidados críticos.

También es de destacar que en la evaluación con LUS de los 12 puntos pulmonares, el hallazgo más común en todos los casos fue la presencia de perfil $B$, lo que sugiere un proceso intersticial como principal mecanismo fisiopatológico de la enfermedad, con baja frecuencia de consolidaciones y derrame pleural. Otros hallazgos secundarios sugieren que la ventilación mecánica y los biomarcadores cardiacos elevados (troponina I y NT-proBNP) también se asocian a resultados adversos. Ninguno de los médicos que realizaron la ecografía resultó infectado, lo que se comprobó mediante dos pruebas virales (identificación en hisopo nasal de ácido nucleico viral por RT-PCR y prueba de anticuerpos IgG).

EI LUS tiene varias ventajas:

- Está disponible en la mayoría de los centros.

- Es de rápida realización.

- La curva del aprendizaje que se requiere es relativamente corta.

- Permite realizar una evaluación de múltiples regiones pulmonares. 
Tabla 3. Modelo de regresión logística múltiple para la predicción de mortalidad

\begin{tabular}{|l|c|c|c|c|}
\hline Variable & HR & EE & p & IC $95 \%$ \\
\hline Edad & 1.02 & 0.01 & 0.12 & $0.99-1.05$ \\
\hline Sexo masculino & 1.08 & 0.43 & 0.84 & $0.49-2.38$ \\
\hline Hipertensión & 1.42 & 0.51 & 0.32 & $0.70-2.90$ \\
\hline Diabetes & 1.02 & 0.36 & 0.94 & $0.51-2.04$ \\
\hline Ventilación mecánica & 3.21 & 1.73 & 0.03 & $1.11-9.27$ \\
\hline Otra infección & 2.09 & 0.94 & 0.10 & $0.86-5.07$ \\
\hline$\geq 19$ líneas B & 2.55 & 0.93 & 0.01 & $1.24-5.23$ \\
\hline Troponina $>20$ pg/mL & 2.16 & 0.75 & 0.02 & $1.09-4.29$ \\
\hline NT-proBNP $>800 \mathrm{pg} / \mathrm{mL}$ & 2.55 & 1.09 & 0.02 & $1.10-5.92$ \\
\hline Dímero D > 600 ng/mL & 1.12 & 0.40 & 0.74 & $0.55-2.26$ \\
\hline Proteína C reactiva > 100 mg/dL & 1.72 & 0.66 & 0.16 & $0.80-3.68$ \\
\hline Ferritina > $700 \mathrm{mg} / \mathrm{dL}$ & 2.09 & 0.98 & 0.11 & $0.833-5.28$ \\
\hline Puntuación RALE > 6 puntos & 1.06 & 0.36 & 0.85 & $0.54-2.07$ \\
\hline
\end{tabular}

$\mathrm{HR}=$ cociente de riesgo (hazard ratio), EE = error estándar, IC $95 \%$ = intervalo de confianza de $95 \%$.

- Limita el riesgo de exposición del personal médico y otro personal de salud.

- Disminuye el movimiento del paciente fuera de la cama de hospitalización.

- Muestra un impacto en la predicción de resultados adversos.

Por lo anterior, consideramos al LUS como una herramienta invaluable en el diagnóstico y seguimiento de los pacientes con COVID-19 y enfermedad del parénquima pulmonar.

\section{Limitaciones}

Este estudio se realizó en un solo centro hospitalario. Por otra parte, se deberá hacer un seguimiento de los pacientes que sobrevivieron, para evaluar la mortalidad, las complicaciones a largo plazo después del alta y el grado de secuelas pulmonares.

\section{Conclusión}

EI LUS es una herramienta clínica de fácil acceso, segura y de rápida realización que se puede llevar a cabo al lado de la cama del paciente. Una puntuación de LUS elevada ayuda a predecir la mortalidad intrahospitalaria.
Proponemos que este estudio se aplique de forma rutinaria en los pacientes con COVID-19 para predecir el desenlace, establecer una terapia agresiva y ayudar en el ingreso temprano a una unidad de cuidados críticos.

\section{Agradecimientos}

Agradecemos al grupo de trabajo de Ultrasonografía de Cuidados Críticos del Instituto Nacional de Cardiología "Ignacio Chávez".

\section{Conflicto de intereses}

Los autores declaran no tener ningún conflicto de intereses.

\section{Financiamiento}

Esta investigación no recibió financiación del sector público, comercial o sin ánimo de lucro.

\section{Responsabilidades éticas}

Protección de sujetos humanos y animales. Los autores declaran que no se realizaron experimentos en humanos 0 animales para este estudio.

Confidencialidad de los datos. Los autores declaran haber seguido los protocolos de su centro de trabajo sobre la publicación de datos de pacientes.

Derecho a la privacidad y consentimiento informado. Los autores declaran que en este artículo no aparecen datos de pacientes.

\section{Bibliografía}

1. Zhang Z, Hou Y, Li D, Li F. Laboratory findings of COVID-19: a systematic review and meta-analysis. Scand J Clin Lab invest. 2020:1-7.

2. Lomoro P, Verde F, Zerboni F, Simonetti I, Borghi C, Fachinetti C, et al. COVID-19 pneumonia manifestations at the admission on chest ultrasound, radiographs, and CT: single-center study and comprehensive radiologic literature review. Eur J Radiol Open. 2020;7:100231.

3. Gopar-Nieto R, Rivas-Lasarte M, Moya-Álvarez A, García-Cruz E, Manzur-Sandoval D, Arias-Mendoza A, et al. Lung ultrasound for the identification of COVID-19 pneumonia. Arch Cardiol Mex. 2020:90:15-18.

4. García-Cruz E, Manzur-Sandoval D, Rascón-Sabido R, Gopar-Nieto R, Barajas-Campos RL, Jordán-Ríos A, et al. Critical care ultrasonography during COVID-19 pandemic: The ORACLE protocol. Echocardiography. 2020;37:1353-1361.

5. Lichtenstein D. Lung ultrasound in the critically ill. Ann Intensive Care. 2014;9:

6. Antúnez-Montes O, Buonsenso D. Routine use of point-of-care lung ultrasound during the COVID-19 pandemic. Medicina Intensiva. 2020 May 07.

7. Peh W, Chan S, Lee Y, Gare P, Ho V. Lung ultrasound in a Singapore COVID-19 intensive care unit patient and a review of its potential clinical utility in pandemic. J Ultrason. 2020;20:e154-e158.

8. Volpicelli G, Lamorte A, Villén T. What's new in lung ultrasound during the COVID-19 pandemic. Intensive Care Med. 2020;46:1445-1448.

9. Warren M, Zhao Z, Koyama T, Batarache JA, Shaver CM, Semler MW, et al. Severity scoring of lung oedema on the chest radiograph is associated with clinical outcomes in ARDS. Thorax. 2018;73:840-846. 